\title{
Molecular Characterization of the Transmission between the Colonization of Methicillin-resistant Staphylococcus aureus to Human and Environmental Contamination in Geriatric Long-term Care Wards
}

\author{
Norichika ASOH****, Hironori MaSAKI*, Hiroshi WatanaBe*, Kiwao Watanabe*, \\ Hiroaki Mitsusima**, Keizou MATSUMOTO**, Kazunori Oishi* and Tsuyoshi NAGATAKE*
}

\begin{abstract}
Objective Transmission between human and environmental contamination from colonized methicillin-resistant Staphylococcus aureus (MRSA) remains a controversial issue. We, therefore, investigated the differences between MRSA types which colonize in humans and in the environment.

Methods A 4-week prospective culture survey for MRSA was performed for 12 patients as well as for the environment of the room of MRSA carriers in quarantine in the geriatric long-term care ward of a 270-bed hospital.
\end{abstract}

Results A total of 97 S. aureus strains (80 MRSA and 17 methicillin-sensitive Staphylococcus aureus [MSSA]) was isolated during the periods of September 8 to 10,23 to 25 and October 5 to 7, 1998; 25 strains were from the respiratory tract, 4 strains from feces and 11 strains from decubitus ulcers. Fifty-seven strains were from the patients' environment. Molecular typing by pulsed-field gel electrophoresis (PFGE) with the Sma I restriction enzyme demonstrated that the predominant type of MRSA isolated from the environment changed by the minute. The patterns of 42 MRSA strains isolated from the environment were identical in $26(61.9 \%)$, closely related in $15(35.7 \%)$ and possibly related in $1(2.4 \%)$ of the cases of those isolated from patients simultaneously. There was no correlation between patients and the environment with the 17 MSSA isolates.

Conclusion Our results demonstrated that MRSA from patients can contaminate the environment, whereas MRSA from the environment might be potentially trans- mitted to patients via health care workers under unsatisfactory infection control.

(Internal Medicine 44: 41-45, 2005)

Key words: Methicillin-resistant Staphylococcus aureus, MRSA, pulsed-field gel electrophoresis, PFGE, environment, geriatric long-term care ward

\section{Introduction}

Methicillin-resistant Staphylococcus aureus (MRSA) is an important pathogen and a major cause of nosocomial infections (1). MRSA strains colonize easily in the host particularly in immunodeficient patients which can cause a variety of serious difficult-to-control infections including septicemia, pneumonia, endocarditis, meningitis and postoperative intra-abdominal infection (2-5). The transmission of MRSA occurs through direct person-to-person contact, usually via the hands of health care workers. However, the prevalence of the infection caused by MRSA remains relatively high despite the intensification of preventive measures (6-8). Of the many possible factors, the most likely factor is poor compliance with recommended infection control policies by health care workers (9). On the other hand, some authors have suggested that contaminated environmental surfaces may serve as a reservoir for MRSA in hospitals (10-18). However, this issue remains a controversial one.

To assess the transmission of MRSA between patients and environmental contamination in a hospital setting, we compared MRSA isolated from patients with that from the envi-

From *Department of Internal Medicine, Institute of Tropical Medicine, Nagasaki University, Nagasaki, **Department of Internal Medicine, Aino Memorial Hospital, Nagasaki and ***Department of Internal Medicine, Juzenkai Hospital, Nagasaki

Received for publication February 17, 2004; Accepted for publication August 17, 2004

Reprint requests should be addressed to Dr. Norichika Asoh, Department of Internal Medicine, Institute of Tropical Medicine, Nagasaki University, 1-12-4 Sakamoto, Nagasaki 852-8523 
ronmental contamination using a molecular technique by pulsed-field gel electrophoresis (PFGE) at an intervals of 2 weeks.

\section{Materials and Methods}

\section{Setting}

Aino Memorial Hospital (AMH), which is affiliated with Nagasaki University, is a 270-bed long-term care facility consisting of 180 beds in internal medicine wards including geriatric medicine and 90 orthopedics beds. The average length of hospital stay in the geriatric wards is approximately 110 days. In this study, an 8-bed cohorting room for seriously affected patients in a geriatric long-term care ward was used for the investigation of possible relationships, including a longitudinal study, between the type of $S$. aureus isolated from patients and that isolated from their hospital room.

\section{Patients}

This study was performed with a total of 12 seriously affected patients ( 5 males and 7 females, mean age, 77.4 years) who had been admitted to AMH on September 8 to 10,23 to 25 and October 5 to 7, 1998. Decubitus ulcers were observed in 4 cases $(33.3 \%)$. In this study interval, no patients became infected with $S$. aureus when infection control measures were used, which included hand washing, disinfection of the upper respiratory tract and decubitus ulcers by treatment with povidone iodine, and cleaning the floor at daily intervals.

\section{Sampling and culture of S. aureus}

Cultures were obtained simultaneously from the nares, pharynx, and rectum with a sterile swab. At the same time, sputum samples were collected using sterile suction tubes. Opened rabbit blood agar plates were placed on the foor of the room of the MRSA carriers in quarantine for 4 or 12 hours in 6 locations throughout the room (4 in each corner, one in the center and one in the entrance). Surveillance was performed three times at 2-week intervals at a specific time. Cultures were performed using TSA II medium (Becton Dickinson, San Jose, CA) supplemented with 5\% rabbit blood agar and $S$. aureus-selective medium (mannitol salt agar, Eiken Chemical Co., Tokyo, Japan) for $18 \mathrm{hrs}$ at $35^{\circ} \mathrm{C}$. The identification of $S$. aureus was based on the morphology of the colonies and the use of specific kits (Staphylo-LA by slide latex agglutination, Denka Seiken Co., Tokyo, Japan). MRSA were identified by the oxacillin disk diffusion method (Kirby-Bauer) according to the guidelines of the National Committee for Clinical Laboratory Standards (19). Ninety-seven strains of $S$. aureus , 40 from patients (7 from nares, 9 from pharynx, 9 from sputum, 4 from feces and 11 from decbitus ulcer) and 57 from hospital environment, were identified.

\section{Pulsed-field gel electrophoresis}

MRSA isolates were grown overnight in Brain Heart
Infusion (BHI) broth at $35^{\circ} \mathrm{C}$, and PFGE by Sma I chromosomal digestion was performed as described previously (20). The DNA was digested with $10 \mathrm{U}$ of SmaI (Takara Shuzo Co., Shiga, Japan) at $30^{\circ} \mathrm{C}$ overnight. A CHEF Mapper pulsed-field electrophoresis system (Bio-Rad Life Science) was used for electrophoresis, with the potential set at 6 $\mathrm{V} / \mathrm{cm}$, switch times set at 0.47 and 63 seconds, and the run time set at 20 hours 18 minutes. After staining with ethidium bromide, the band patterns were compared according to the criteria for bacterial strain typing, as described by Tenover et al (21).

\section{Results}

\section{Cultures of patients}

Each of the 24 cultures in the nares, pharynx, sputum and faeces and 14 cultures in the decubitus ulcer revealed that 7 $(29.2 \%)$ in the nares, $9(37.5 \%)$ in the pharynx, $9(37.5 \%)$ in the sputum, $4(16.7 \%)$ in the faeces and $11(78.6 \%)$ in the decubitus ulcer were contaminated with $S$. aureus. Thirtyeight $(95 \%)$ of these 40 strains were determined to be MRSA.

\section{Cultures of environment surface}

Forty $(44.4 \%)$ of 90 environment surfaces were contaminated with $S$. aureus. Eight cultures yielded fewer than ten colonies, 27 cultures yielded from ten to ninty-nine colonies and the remaining 5 cultures yielded more than one hundred colonies of $S$. aureus. In addition, one strain of $S$. aureus in 26 plates, two strains of those in 11 plates and three strains of those in 3 plates were examined for difference in colonial characteristics. Therefore, a total of 57 environment surface cultures yielded S. aureus. Forty-two (73.7\%) of these 57 strains were determined to be MRSA.

\section{Interpretation of PFGE}

The total of 97 strains of $S$. aureus (80 MRSA) were analyzed by PFGE (Fig. 1). Molecular typing demonstrated that the patterns of the 42 MRSA isolates from the environment were identical in $26(61.9 \%)$, closely related in $15(35.7 \%)$ and possibly related in $1(2.4 \%)$ with 38 MRSA isolated from the patients simultaneously, despite the fact that no correlation between human and environment in 17 methicillinsensitive S. aureus (MSSA) was found (Table 1). The genotypes of $S$. aureus isolated from the environment changed by the minute even if they were in same area on September 8 to 10, 23 to 25 and October 5 to 7, 1998, respectively (Table 2 ).

\section{Discussion}

Some previous reports have suggested that environmental reservoirs might be a potential route of transmission for epidemic strains (22-24). However, others have concluded that the environment may not be important because of differences in the phenotypes in $S$. aureus isolated from the hospital 


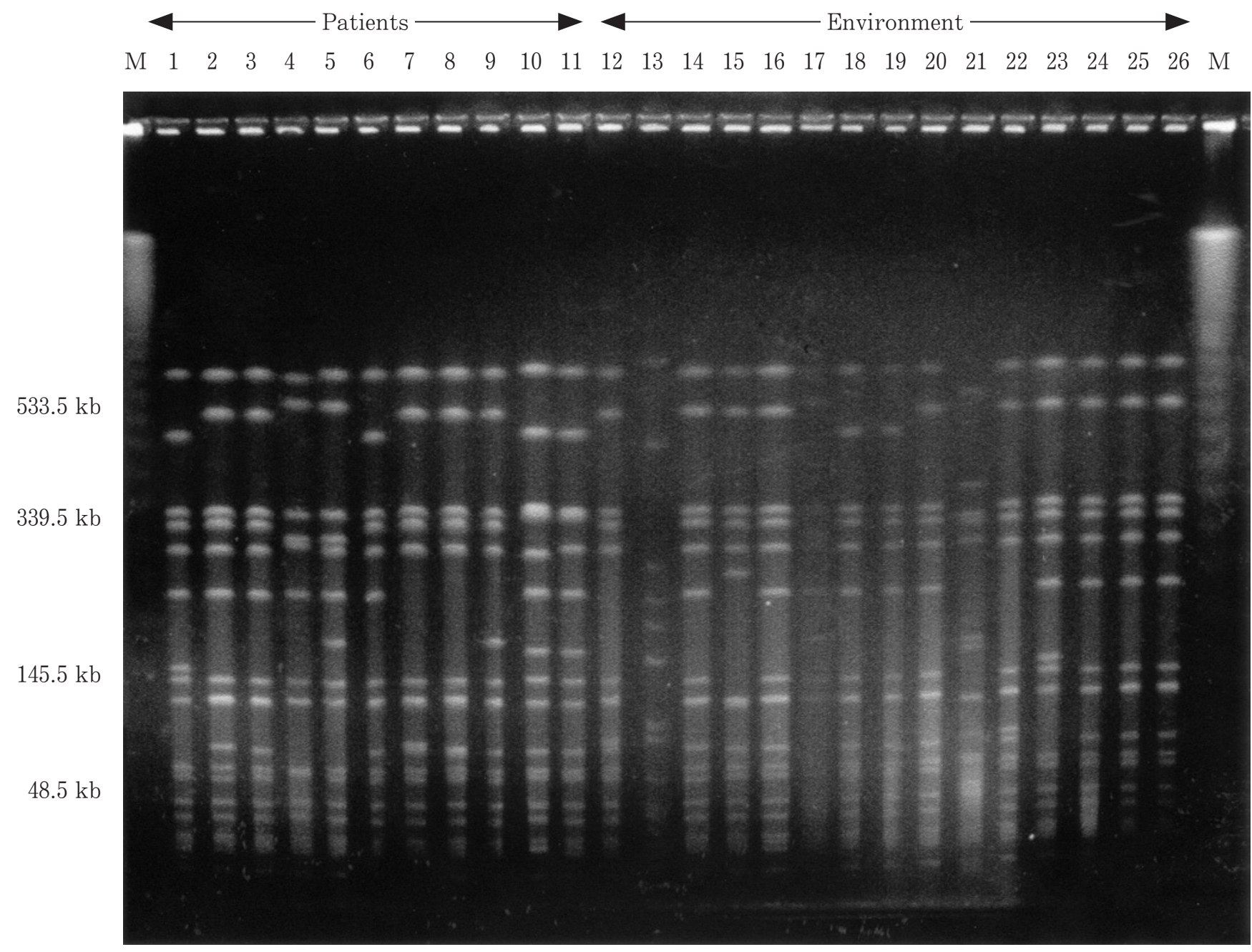

PFGE pattern A1 A2 A2 A3 A4 A5 A6 A6 A7 A8 A8

A6 B1 A2 A9 A2 A4 A5 A5 A2 C1 A6 A10 A2 A2 A2

Figure 1. Pulsed-field gel electrophoresis (PFGE) patterns of Sma I digested DNA of S. aureus isolated from patients and the environment. Lanes 1-11, isolated from patients; lanes 12-26, isolateed from the environment on September 8 to 10, 1998. M indicates molecular weight markers (lambda ladder DNA size standards; Bio-Rad Laboratories). Of these 26 lanes, lanes 13 and 21 were methicillin-sensitive $S$. aureus (MSSA). The other lanes were Methicillin-resistant Staphylococcus aureus (MRSA). The genotype of the MRSA between patients and environment is identical (lanes 2, 3, 14, 16, 20, 24-26; 5, 17; 6, 18, 19; 7, 8, 12, 22) or closely related.

environment and patients $(25,26)$. Recently, the association between the dispersion of MRSA strains into the environment by colonized patients and MRSA epidemics has been increasingly reported via the use of molecular typing methods (10-18). However, the transmission between colonies of MRSA to human and environmental contamination continues to be a controversial issue and remains uncertain. In the present study, we examined the relationship between the environment and seriously affected elderly patients who had some risk factors for MRSA infection (27), such as bedridden status, and the presence of decubitus ulcers. In particular, we repeatedly examined environmental cultures, whereas previous studies have investigated these only on a temporary basis. Our molecular analysis demonstrates that the DNA patterns of MRSA from the environment were identical or closely related to those from patients in a geriatric long-term ward, and the predominant type of MRSA isolated from the environment changed by the minute under conditions of intensified preventive measures including cleaning the floors. In our hospital, we have achieved a significant reduction of MRSA bacteremia in geriatric wards after the introduction of infection control measures against nosocomial infections (28), and these infection control measures have continued to the present time. During this study period, MRSA infections, including bacteremia, did not occur despite the isolation of MRSA strains from the environment. 
AsOH et al

Table 1. Genetic Relationships of MRSA Strains Isolated from Patients and the Hospital Environment

\begin{tabular}{lcccc}
\hline & \multicolumn{4}{c}{ No. of environment } \\
\cline { 2 - 4 } PFGE & $\begin{array}{c}\text { September 8 to 10. } \\
\text { interpretation }\end{array}$ & $\begin{array}{c}\text { September 23 to 25. } \\
\text { Total (\%) }\end{array}$ & $\begin{array}{c}\text { October } 5 \text { to } 7 . \\
1998\end{array}$ & \\
\hline Identical & 1998 & 1998 & 1 & $26(61.9)$ \\
Closely related & 15 & 12 & 1 & $15(35.7)$ \\
Possibly related & 2 & 1 & 0 & $1(2.4)$ \\
Different & 0 & 0 & 0 & $0(0)$ \\
\hline \multicolumn{1}{c}{ Total } & 17 & 23 & 2 & 42 \\
\hline
\end{tabular}

Table 2. Distribution of PFGE Patterns of S. aureus Isolated from Each Area on September 23 to 25

\begin{tabular}{|c|c|c|c|c|c|c|}
\hline & Area. 1 & Area. 2 & Area. 3 & Area. 4 & Area. 5 & Area. 6 \\
\hline Period 1 & & & $\begin{array}{l}\text { MRSA (A12) } \\
\text { MRSA (A13) }\end{array}$ & $\begin{array}{l}\text { MRSA (A12) } \\
\text { MRSA (A13) }\end{array}$ & & MRSA (A8) \\
\hline Period 2 & MRSA (A12) & $\begin{array}{l}\text { MRSA (A8) } \\
\text { MRSA (A8) }\end{array}$ & MRSA (A11) & $\begin{array}{l}\text { MSSA (D) } \\
\text { MRSA (A2) }\end{array}$ & $\begin{array}{l}\text { MRSA (A8) } \\
\text { MRSA (A8) } \\
\text { MRSA (A8) }\end{array}$ & $\begin{array}{c}\text { MRSA (A8) } \\
\operatorname{MSSA}(\mathrm{K})\end{array}$ \\
\hline Period 3 & $\operatorname{MSSA}(\mathrm{J})$ & & & & & \\
\hline Period 4 & & & & MRSA (A8) & MRSA (A2) & \\
\hline Period 5 & & & $\begin{array}{c}\text { MSSA (E) } \\
\text { MRSA (A12) }\end{array}$ & $\begin{array}{l}\text { MRSA (A8) } \\
\text { MRSA (A14) } \\
\text { MRSA (A16) }\end{array}$ & $\begin{array}{l}\text { MRSA (A15) } \\
\text { MRSA (A15) }\end{array}$ & MRSA (A8) \\
\hline
\end{tabular}

Period 1: 8 PM on September 23-8 AM on September 24, Period 2: 8 AM on September 24-12 AM on September 24, Period 3: 12 AM on September 24-4 PM on September 24, Period 4: 4 PM on September 24-8 PM on September 24, Period 5: 8 PM on September 24-8 AM on September 25.

In conclusion, our results demonstrated that MRSA from patients can contaminate the environment, whereas MRSA from the environment might be potentially transmitted to patients via health care workers under unsatisfactory infection control.

Acknowledgements: We wish to thank Akihiro Wada (Department of Bacteriology, Institute of Tropical Medicine, Nagasaki University), Chieko Shimauchi (Miyazaki Prefectural Nursing University) and Matsuhisa Inoue (Kitasato University School of Medicine) for their help in completing the PFGE studies.

\section{References}

1) Wenzel RP, Nettleman MD, Jones RN, Pfaller MA. Methicillinresistant Staphylococcus aureus: implications for the 1990s and effective control measures. Am J Med 91 (suppl 3B): 221S-227S, 1991.

2) Rubio M, Romero J, Corral O, Roca V, Picazo JJ. Bacteremia by Staphylococcus aureus: analysis of 311 episodes. Enferm Infec Microbiol Clin 17: 56-64, 1999.

3) Gonzalez C, Rubio M, Romero-Vivas J, Gonzalez M, Picazo JJ. Bacteremic pneumonia due to Staphylococcus aureus: A comparison of disease caused by methicillin-resistant and methicillin-susceptible organisms. Clin Infect Dis 29: 1171-1177, 1999.

4) Sakamoto T, Kikuchi K, Mineura K, Kowada M, Nakagomi O. MRSA meningitis in postoperative patients. Report of 4 cases. Jpn J Antibiot 43: $1137-1142,1990$.

5) Fierobe L, Decre D, Muller C, et al. Methicillin-resistant Staphylococcus aureus as a causative agent of postoperative intra-abdominal infection: relation to nasal colonization. Clin Infect Dis 29: 1231-1238, 1999.

6) Boyce JM. Strategies for controlling methicillin-resistant Staphylococcus aureus in hospitals. J Chemother 7 (suppl 3): 81-85, 1995.

7) Vandenbroucke-Grauls CM. Methicillin-resistant Staphylococcus aureus control in hospitals: the Dutch experience. Infect Control Hosp Epidemiol 17: 512-513, 1996.

8) Speller DC, Johnson AP, James D, Marples RR, Charlett A, George RC. Resistance to methicillin and other antibiotics in isolates of Staphylococcus aureus from blood and cerebrospinal fluid, England and Wales, 1989-95. Lancet 350: 323-325, 1997.

9) Pettinger A, Nettleman MD. Epidemiology of isolation precautions. Infect Control Hosp Epidemiol 12: 303-307, 1991.

10) Boyce JM, Potter-Bynoe G, Chenevert C, King T. Environmental contamination due to Methicillin-resistant Staphylococcus aureus: possible infection control implications. Infect Control Hosp Epidemiol 18: 622627, 1997. 


\section{MRSA and Environment}

11) Wagenvoort JHT, Davies BI, Westermann EJ, Werink TJ, Toenbreker HM. MRSA from air-exhaust channels. Lancet 341: 840-841, 1993.

12) Blythe D, Keenlyside D, Dawson SJ, Galloway A. Environmental contamination due to methicillin-resistant Staphylococcus aureus (MRSA). J Hosp Infect 38: 67-69, 1998.

13) Oie S, Kamiya A. Contamination of environmental surfaces by methicillin-resistant Staphylococcus aureus (MRSA). Biomedical Letters 57: 115-119, 1998.

14) Stacey A, Burden P, Croton C, Jones E. Contamination of television sets by methicillin-resistant Staphylococcus aureus (MRSA). J Hosp Infect 39: 243-244, 1998.

15) Kumari DN, Haji TC, Keer V, Hawkey PM, Duncanson V, Flower E. Ventilation grilles as a potential source of methicillin-resistant Staphylococcus aureus causing an outbreak in an orthopaedic ward at a district general hospital. J Hosp Infect 39: 127-133, 1998.

16) Farrington $M$, Ling J, Ling T, French GL. Outbreaks of infection with methicillin-resistant Staphylococcus aureus on neonatal and burns units of a new hospital. Epidemiol Infect 105: 215-228, 1990.

17) Lessing MP, Jordens JZ, Bowler IC. Molecular epidemiology of a multiple strain outbreak of methicillin-resistant Staphylococcus aureus amongst patients and staff. J Hosp Infect 31: 253-260, 1995.

18) Shiomori T, Miyamoto H, Makishima K. Significance of airborne transmission of methicillin-resistant Staphylococcus aureus in an otolaryngology-head and neck surgery unit. Arch Otolaryngol Head Neck Surg 127: 644-648, 2001.

19) National Committee for Clinical Laboratory Standards. Performance standards for antimicrobial disk susceptibility tests. Approved standard. NCCLS document M2-A6. National Committee for Clinical Laboratory Standards, Wayne, Pa, 1998.

20) Watanabe $\mathrm{H}$, Masaki $\mathrm{H}$, Asoh $\mathrm{N}$, et al. Molecular analysis of methicillin-resistant Staphylococcus aureus as a causatine agent of bronchopulmonary infection: Relation to colonization in the upper respiratory tract. J Clin Microbiol 38: 3867-3869, 2000.

21) Tenover FC, Arbeit RD, Goering RV, et al. Interpreting chromosomal DNA restriction patterns produced by pulsed-field gel electrophoresis: criteria for bacterial strain typing. J Clin Microbiol 33: 2233-2239, 1995.

22) Rahman M. Epidemic methicillin-resistant Staphylococcus aureus (EMRSA): experience from a health district of central England over five years. Postgrad Med J 69 (Suppl 3): S126-S129, 1993.

23) Layton MC, Perez M, Heald P, Patterson JE. An outbreak of mupirocin-resistant Staphylococcus aureus on a dermatology ward associated with an environmental reservoir. Infect Control Hosp Epidemiol 14: 369-375, 1993.

24) Masaki H, Asoh N, Watanabe $H$, et al. Possible relationship between Staphlococcus aureus colonizing the respiratory tract and rectum and $\mathrm{S}$. aureus isolated in a geriatric hospital environment. Intern Med 42: $281-$ 282, 2003.

25) Bradley SF, Terpenning MS, Ramsey MA, et al. Methicillin-resistant Staphylococcus aureus: Colonization and infection in a long-term care facility. Ann Intern Med 115: 417-422, 1991.

26) Maki DG, Alvarado CJ, Hassemer CA, Zilz MA. Relation of the inanimate hospital environment to endemic nosocomial infection. N Engl J Med 307: 1562-1566, 1982.

27) Bradley SF. Methicillin-resistant Staphylococcus aureus: long-term care concerns. Am J Med 106: 2S-10S, 1999.

28) Masaki H, Watanabe H, Degawa S, et al. Significant reduction of methicillin-resistant Staphylococcus aureus bacteremia in geriatric wards after introduction of infection control measures against nosocomial infections. Intern Med 40: 214-220, 2001. 